\title{
Impact de trois techniques de restauration des sols sur la survie et la croissance de trois espèces ligneuses sur les «zipellés » au Burkina Faso
}

\author{
François Wenemi KAGAMBEGA*, Salifou TRAORE, Adjima THIOMBIANO et \\ Joseph Issaka BOUSSIM
}

Université de Ouagadougou, Unité de Formation et de Recherche en Sciences de la vie et de la Terre, Laboratoire de Biologie et Ecologie Végétales, 09 BP 848 Ouagadougou 09, Burkina Faso.

*Auteur correspondant, E-mail: wenemifrancois@yahoo.fr, Tel: +22670747578; +22678035989

\section{RESUME}

A la lumière des phénomènes de dégradation des sols, un essai de régénération artificielle d'espèces ligneuses utilisant des technologies d'aménagement a été conduit dans la zone soudano-sahélienne du Burkina Faso. Il avait pour objectif d'appréhender l'impact des techniques de restauration des sols (demi-lunes, soussolage et amendement) sur les performances de Combretum micranthum, Jatropha curcas et Faidherbia albida à Gampéla. Un dispositif en blocs randomisés combinant les plantations des trois espèces avec les aménagements a été utilisé sur un sol nu et encroûté. Les résultats après la première saison pluvieuse indiquent que les aménagements ont influencé de manière significative ( $\mathrm{p}<0,0001)$ la croissance des plants à l'exception de $F$. albida $(\mathrm{p}=0,627)$. Au bout de 2 ans, la croissance et les taux de survie ont été nettement améliorés dans les aménagements de demi-lunes et dans une moindre mesure de sous solage avec des valeurs de survie élevées (> 70\% dans les demi-lunes chez tous les espèces) et de croissance atteignant parfois le double des témoins. Cet impact positif est imputable à l'amélioration des conditions du sol (compacité, fertilité et humidité) induite par les différentes techniques.

(C) 2011 International Formulae Group. All rights reserved.

Mots clés: Dégradation des sols, afforestation, réhabilitation, érosion, Burkina Faso.

\section{INTRODUCTION}

La dégradation des terres qui se traduit par une perte de la productivité des sols met en péril les moyens de subsistance d'un milliard de personnes dans le monde (FEMFIDA, 2002). Cette dégradation est le résultat d'un ensemble de facteurs dont la sécheresse, les pratiques agricoles et les aménagements forestiers non durables. Ce phénomène affecte 20 à $60 \%$ des terres en Afrique (Thiombiano et Tourino-Soto, 2007), entraînant une perte annuelle de 6 millions d'hectares de terres productives, soit 42 milliards de dollars US évalués en termes de diminution des recettes due à la dégradation des terres (UNDP-GEF, 2004). Il en résulte des impacts négatifs sur la sécurité alimentaire et la lutte contre la pauvreté. Plus de la moitié de la population de l'Afrique est affectée par les processus de dégradation, faisant d'elle l'un des continents dont les issues urgentes de développement nécessitent des investissements considérables (Bationo et al., 2006). La restauration des terres dégradées est donc une priorité urgente 
pour l'équilibre environnemental et climatique mondial (Lal, 2004). Les régions semi-arides et arides offrent des potentialités importantes à travers la reforestation et la restauration du couvert végétal des terres dégradées (Dixon et al., 1993; Bellefontaine et al., 2005). Selon le protocole de Kyoto, l'afforestation des terres dégradées offre des potentialités à augmenter significativement la séquestration du carbone et à mitiger le phénomène de dégradation. Cependant, l'afforestation des terres dégradées est difficilement réalisable sans des techniques d'aménagement adéquates. $\mathrm{Au}$ stade ultime de cette dégradation, les terres sont dénudées, quasi imperméables et stériles: «zipellés» en langue locale moré. Ces zipellés qui représentent $24 \%$ des terres arables du Burkina Faso (Zougmoré et al., 2003) sont caractérisés par leurs fortes compacités et faible niveau de fertilité pour permettre une régénération naturelle (Hien, 1995; Ambouta et al., 1996). Afin d'améliorer les conditions de ces milieux, en particulier l'infiltration de l'eau, l'enracinement et la nutrition, diverses techniques de conservation des eaux et des sols (CES) sont utilisées avec succès dans les terres à vocation agricole (Roose, 2004; Zougmoré et al., 2004) dont les demi-lunes, le sous-solage et l'amendement. Ces techniques ont été peu appliquées dans les terres à vocation forestière (Ganaba et al., 2006). Toutefois, les études réalisées étaient surtout des évaluations à la suite d'intervention de projets de développement; ainsi les variabilités des types d'utilisation fonctionnelle des terres et des sols ne permettaient que des comparaisons limitées (Ganaba, 2005). L'effet de ces techniques sur la régénération artificielle des espèces ligneuses doit être largement exploré pour des perspectives de reforestation de terres dégradées. Le succès participatif de cette reforestation exige à la fois la sélection d'espèces locales résistantes aux stress hydriques (Traore et al., 2007; Kumar et al., 2008) et utilitaires pour les populations. Sur ces zipellés caractérisés par une rareté ou une absence de régénération naturelle après une saison sèche, comment ces ouvrages affectentils la croissance et la survie des espèces ?

La présente étude a ainsi pour objectif d'appréhender l'impact de ces trois techniques de CES sur la croissance et le taux de survie de trois espèces utilitaires en zone nord soudanienne du Burkina Faso. Elle se fonde sur les deux hypothèses suivantes: (i) les techniques d'aménagement améliorent considérablement la croissance et le taux de survie des espèces ligneuses. (ii) ces performances de régénération au niveau de chaque espèce varient significativement en fonction des différentes techniques de CES.

\section{MATERIEL ET METHODES \\ Le Milieu d'étude}

L'étude a été menée à Gampéla $\left(12^{\circ}\right.$ $\left.25^{\prime} \mathrm{N} ; 1^{\circ} 21^{\prime}, \mathrm{W}\right)$ situé à $20 \mathrm{~km}$ de Ouagadougou, au centre du Burkina Faso (Figure 1). Sur le plan phytogéographique, Gampéla appartient au secteur nord-soudanien (Fontes et Guinko, 1995). Il est soumis à un régime pluviométrique uni-modal, allant de mai à octobre. Ce régime pluviométrique est marqué par une irrégularité spatio-temporelle avec une valeur moyenne interannuelle sur la période 1980-2009 de $760 \mathrm{~mm}$. La température moyenne varie entre $23{ }^{\circ} \mathrm{C}$ en décembre-janvier et $35{ }^{\circ} \mathrm{C}$ en avril-mai. Les différents types d'états de surface rencontrés sur le site d'étude sont généralement constitués de croûtes d'érosion. Les sols sont des Glevic-Luvisols (FAO, 2006). Il s'agit de sols limono-argileux totalement dénudés avec une pente moyenne de 1,5\%; les propriétés physico-chimiques (Tableau 1) révèlent qu'ils sont acides, pauvres en carbone et azote organiques, en phosphore assimilable et en potassium disponible avec de faibles capacité d'échange cationique et somme de bases échangeables. 


\section{Le matériel végétal}

Trois espèces végétales ont été choisies: Faidherbia albida (Del.) A., Combretum micranthum G. Don. Chev. et Jatropha curcas L. Elles ont été choisies pour leurs nombreux avantages aussi bien écologiques que socio-économiques.

F. albida appartient à la famille des Mimosaceae qui de part leur habilité à fixer l'azote atmosphérique et leur symbiose avec les Rhizobiaceae, contribuent à l'amélioration de la productivité des sols (Pule-Meuelenberg et Dakora, 2005). En plus de cela, l'espèce procure divers services aux populations rurales parmi lesquels le bois et le fourrage. Très répandue en Afrique subsaharienne sèche, $F$. albida se développe de préférence dans les plaines alluviales (Alexandre et Ouédraogo, 1993).

C. micranthum (Combretaceae) est fortement utilisée par la population dans la médecine traditionnelle ainsi qu'en artisanat dans la confection de meubles et d'objets de ménage; sur le plan écologique, l'espèce affectionne les sols argilo-sableux superficiels (Thiombiano, 2005).

Espèce introduite, J. curcas (Euphorbiaceae) est utilisée par les populations dans la médecine traditionnelle et comme haie vive (Assogbadjo et al., 2009). Réputée pour sa résistance à la sécheresse et sa grande capacité d'adaptation à tous les types de sols, l'espèce a connu ces dernières années un regain d'intérêt à cause de son utilisation comme biocarburant.

\section{Le dispositif expérimental}

Une pépinière a été installée en miavril 2008 à Ouagadougou. Les semences des trois espèces ont été semées dans des pots en plastique (mesurant $7.5 \mathrm{~cm} \times 25.5 \mathrm{~cm}$ à vide) remplis d'un mélange de sable, d'argile et de fumier de petits ruminants dans les proportions volumiques respectives de 1: $2: 1$. Le substrat a été gardé humide durant la production des plants. Aucun fertilisant chimique n'a été utilisé. A la fin de la production (trois mois après), après une sélection, les plants ont été transplantés le 21 juillet 2008 de façon manuelle dans des trous. Le dispositif expérimental, en trois blocs entièrement randomisés (Figure 2) a combiné les plantations de chaque espèce avec les quatre traitements comme suit:

T0: plantation sans aménagement (témoin); A: plantation dans des trous amendés; SL: plantation dans des aménagements de sous-solage non-amendés ;

DL: plantation dans des aménagements de demi-lunes non-amendés

Réalisées manuellement, les demilunes en forme de demi-cercles ont un diamètre de $2 \mathrm{~m}$ et une profondeur comprise entre 10 et $15 \mathrm{~cm}$. Disposées en quinconces perpendiculairement à la pente, l'espacement entre deux demi-lunes sur la même ligne est de $1 \mathrm{~m}$ et de $3 \mathrm{~m}$ entre deux lignes consécutives. Le sous-solage a permis de réaliser des sillons d'une profondeur de 40-60 $\mathrm{cm}$, espacés de $3 \mathrm{~m}$ à l'aide d'un tracteur. La pratique d'amendement a consisté à déposer une poignée $(300 \mathrm{~g})$ de déjection de petits ruminants dans les trous de plantation.

Chaque bloc abritait un total de 12 parcelles correspondant aux combinaisons de plantations des trois espèces avec les aménagements et leurs témoins. Dans chaque parcelle aménagée ou non, 20 plants sont mis en place dans des trous espacés de $3 \mathrm{~m}$ et ayant $50 \mathrm{~cm}$ de diamètre et de profondeur.

\section{Collecte et analyse des données Propriété hydrique du sol}

L'humidité pondérale du sol a été mesurée par la méthode gravimétrique en milieu de saison pluvieuse (août) et en début de saison sèche (octobre). Les échantillons de sols ont été prélevés à cinq niveaux $(0-10 \mathrm{~cm}$, $10-20 \mathrm{~cm}, 20-30 \mathrm{~cm}, 30-40 \mathrm{~cm}$ et $40-50 \mathrm{~cm}$ ). A chaque mesure, cinq trous de prélèvements sont placés de façon aléatoire dans les différentes parcelles aménagées ou non. Les 
prélèvements sont effectués aux abords des trous de plantations.

\section{Performance des espèces plantées}

Dans chaque parcelle, des mesures mensuelles de la hauteur, du diamètre au collet et de la survie des plants ont été réalisées depuis le début de l'essai. Pour les individus multicaules, on a mesuré la hauteur de la tige axiale la plus grosse du ras du sol jusqu'à l'apex.

\section{Analyses statistiques}

Les taux d'humidité du sol, les accroissements et les taux de survie des plants ont été analysés par le modèle linéaire généralisé avec des erreurs de type binomial. Ces données remplissent les postulats de normalité et d'homogénéité de variance. Le modèle linéaire généralisé avec des erreurs de type binomial a été utilisé pour pallier la distribution non-normale des erreurs et l'inconstance de la variance qui sont associées aux données binaires. Les variables telles l'année, l'aménagement et la profondeur ont été traitées comme des facteurs catégoriques fixes. Le caractère significatif ou non a été évalué par l'application du test de Tukey HSD pour le classement des moyennes. Les analyses ont été réalisées avec JMP 7 (SAS, 2007).

\section{RESULTATS}

Effets des aménagements sur l'humidité du sol

L'humidité du sol varie significativement à travers les années, les aménagements et les niveaux de profondeur (Tableau 2). Ainsi, suivant les niveaux de profondeurs, les aménagements influencent significativement le taux d'humidité aussi bien en milieu de saison pluvieuse (août) qu'en début de saison sèche (fin octobre).

Dans toutes les parcelles, il existe des différences significatives de stock hydrique en fonction des aménagements et suivant les niveaux de profondeurs (Figure 3). En août, le taux d'humidité diminue généralement de 0 à $30 \mathrm{~cm}$ puis devient constant; cependant en Octobre, le stock hydrique augmente avec la profondeur de 0 à $30 \mathrm{~cm}$ pour se stabiliser ensuite. Considérant les données de 2008, la comparaison entre aménagements montre des valeurs significativement plus élevées dans les DL et le SL $(F=337,84$; $p<0,0001)$. En 2009, les aménagements de DL et SL se distinguent encore avec toutefois des valeurs plus élevées dans les DL surtout en octobre.

\section{Effets des aménagements sur la survie et la croissance des espèces}

L'évolution temporelle de la survie des plants (Figure 4) varie suivant les espèces et est influencée par les aménagements. Pour $J$. curcas, les taux de survie sont presque similaires et élevés dans tous les aménagements jusqu'au début de la saison sèche (octobre-janvier). Par la suite, d'importantes variations inter-aménagements sont observées jusqu'à la fin de l'expérience. A l'exception de l'aménagement de DL, les taux de survie diminuaient fortement dans le SL et encore plus au niveau des autres aménagements (T0 et surtout $\mathrm{A}$ jusqu'à $0 \%$ ). Chez C. micranthum, les taux diminuaient faiblement jusqu'en janvier 2008; à partir de ce moment, pendant que les taux se maintenaient constant et élevés dans les DL $(90 \%)$ et le SL $(80 \%)$, ils diminuaient considérablement dans les autres aménagements (A et T0). Pour F. albida à l'exception de l'aménagement de DL chez lequel les taux étaient élevés et constant, dans les autres aménagements ils diminuaient fortement et constamment dans le temps.

La variation temporelle de la hauteur des plants suit également le rythme des saisons et est influencée tout de même par les aménagements (Figure 5). A la fin de la première saison pluvieuse (octobre 2008), à l'exception de $F$. albida $(\mathrm{p}=0,627)$, les résultats montrent une influence significative des aménagements sur les taux de croissance 
des espèces (Tableau 3). Pour J. curcas, c'est l'amendement (A) qui induit le plus fort accroissement suivi par le sous-solage (SL). Pour $C$. micranthum, c'est le SL, suivi par les DL puis le témoin et l'amendement. Après cette période, d'octobre 2008 jusqu'en juillet 2009, chez toutes les espèces, la hauteur des plants augmentait plus rapidement dans l'aménagement de DL suivi généralement de celui de SL à l'exception de leur apparente similarité observée chez $C$. micranthum; les plus faibles valeurs sont observées dans les aménagements T0 et A. A partir de juillet 2009, l'évolution suit les mêmes tendances jusqu'à la fin de l'essai avec toutefois une baisse de la vitesse de croissance. La Figure 6 montre une corrélation significativement positive entre les taux de croissance en hauteur et diamètre chez les trois espèces et met en évidence les meilleures performances de l'aménagement de DL.

En fin d'expérimentation, l'analyse de variance des taux de croissance et de survie, montre une influence significative des aménagements (Tableau 4). Toutefois, la comparaison des moyennes indique des valeurs de hauteurs, de diamètres et de taux de survie significativement plus élevées dans les DL chez toutes les espèces exceptée la similarité avec SL pour C. micranthum et les plus faibles dans les aménagements $\mathrm{T} 0$ et $\mathrm{A}$ (Figures 4, 5 et 6).

Tableau 1 : Caractéristiques physicochimiques des sols du site expérimental.

\begin{tabular}{lcc}
\hline \multirow{2}{*}{ Paramètres } & \multicolumn{2}{c}{ Profile } \\
\cline { 2 - 3 } & Horizon A & Horizon B \\
\hline Argile (\%) & $15,83 \pm 0,52$ & $42,83 \pm 0,63$ \\
Limon fin (\%) & $9,92 \pm 1,38$ & $12,58 \pm 2,04$ \\
Limon grossier (\%) & $33,83 \pm 2,76$ & $33,51 \pm 1,33$ \\
Sable fin (\%) & $18,16 \pm 2,48$ & $6,76 \pm 0,46$ \\
Sable grossier (\%) & $21,8 \pm 1,16$ & $3,99 \pm 0,93$ \\
Réserve utile en eau (\%) & $8,76 \pm 0,84$ & $11,61 \pm 91$ \\
pH $\left(\mathrm{H}_{2} \mathrm{O}\right)$ & $5,43 \pm 0,39$ & $6,47 \pm 1,16$ \\
pH $(\mathrm{KCl})$ & $4,37 \pm 0,1$ & $5,62 \pm 1,2$ \\
Carbone total $(\mathrm{g} / \mathrm{kg})$ & $4,8 \pm 0,2$ & $3,6 \pm 1,2$ \\
Azote total $(\mathrm{g} / \mathrm{kg})$ & $0,4 \pm 0,1$ & $0,3 \pm 0,1$ \\
Phosphore assimilable $(\mathrm{cmol} / \mathrm{kg})$ & $2,35 \pm 0,81$ & $2,23 \pm 0,82$ \\
Potassium disponible $(\mathrm{cmol} / \mathrm{kg})$ & $23,3 \pm 5,54$ & $21,7 \pm 2,43$ \\
Somme de bases échangeables $(\mathrm{cmol} / \mathrm{kg})$ & $3,27 \pm 1,08$ & $6,75 \pm 0,79$ \\
Capacité d'échange cationique $(\mathrm{cmol} / \mathrm{kg})$ & $4,9 \pm 0,72$ & $9,38 \pm 0,59$ \\
\hline Moyenne \pm erreurs standards; $\mathrm{n}=5$ & &
\end{tabular}

Tableau 2 : Résultats de l'analyse de variance de l'humidité en fonction des différents facteurs et de leur combinaison.

\begin{tabular}{lcccccc}
\hline \multirow{2}{*}{ Facteurs } & \multicolumn{3}{c}{ Août } & \multicolumn{3}{c}{ Octobre } \\
\cline { 2 - 7 } & $\mathrm{dl}$ & $\mathrm{F}$ & $\mathrm{P}$ & $\mathrm{dl}$ & $\mathrm{F}$ & $\mathrm{P}$ \\
\hline Année & 1 & 94,187 & $<0,0001$ & 1 & 3,5194 & 0,0643 \\
Niveau & 4 & 20,475 & $<0,0001$ & 4 & 98,7267 & $<0,0001$ \\
Traitement & 3 & 337,849 & $<0,0001$ & 3 & 279,0492 & $<0,0001$ \\
\hline
\end{tabular}


Tableau 3 : Effets des aménagements sur les accroissements relatifs pendant la saison pluvieuse des espèces.

\begin{tabular}{ccccccc}
\hline & \multicolumn{2}{c}{ J. curcas } & \multicolumn{2}{c}{ C. micranthum } & \multicolumn{2}{c}{$\boldsymbol{F}$ albida } \\
\cline { 2 - 7 } & $\mathrm{H}(\%)$ & $\mathrm{D}(\%)$ & $\mathrm{H}(\%)$ & $\mathrm{D}(\%)$ & $\mathrm{H}(\%)$ & $\mathrm{D}(\%)$ \\
\hline T0 & $20,1 \pm 4,9 \mathrm{c}$ & $139,5 \pm 20,3 \mathrm{~b}$ & $61,5 \pm 11,6 \mathrm{c}$ & $129,3 \pm 22 \mathrm{a}$ & $2,9 \pm 0,8 \mathrm{a}$ & $35 \pm 2,2 \mathrm{a}$ \\
$\mathrm{A}$ & $42,6 \pm 6,6 \mathrm{a}$ & $196,4 \pm 21,3 \mathrm{a}$ & $63,2 \pm 8,3 \mathrm{c}$ & $129,3 \pm 23,1 \mathrm{a}$ & $2,61 \pm 1,1 \mathrm{a}$ & $35,8 \pm 2,9 \mathrm{a}$ \\
SL & $26,6 \pm 6,6 \mathrm{~b}$ & $174,2 \pm 9,1 \mathrm{a}$ & $95,8 \pm 11,9 \mathrm{~b}$ & $190,6 \pm 12,2 \mathrm{a}$ & $2,4 \pm 0,8 \mathrm{a}$ & $30,1 \pm 1,7 \mathrm{a}$ \\
DL & $19,7 \pm 4 \mathrm{c}$ & $138,2 \pm 16,9 \mathrm{~b}$ & $80,6 \pm 13,6 \mathrm{~b}$ & $152 \pm 31,2 \mathrm{a}$ & $2,2 \pm 0,7 \mathrm{a}$ & $28,8 \pm 4,1 \mathrm{a}$ \\
\hline \multicolumn{2}{c}{ ET $=$ Ecart type, $\mathrm{H}=$ hauteur, D $=$ diamètre, Moyenne \pm ET. }
\end{tabular}

Tableau 4 : Résultats de l'analyse de variance avec les taux de survie et de croissance à la fin de l'essai comme variables dépendantes, le type d'espèce et le traitement comme facteurs fixés.

\begin{tabular}{lcccccccc}
\hline \multirow{2}{*}{$\begin{array}{l}\text { Sources de } \\
\text { variation }\end{array}$} & \multicolumn{3}{c}{ Taux de survie (\%) } & \multicolumn{3}{c}{ Diamètre (\%) } & \multicolumn{2}{c}{ Hauteur (\%) } \\
\cline { 2 - 9 } & $\mathrm{dl}$ & $\mathrm{F}$ & $\mathrm{P}$ & $\mathrm{dl}$ & $\mathrm{F}$ & $\mathrm{P}$ & $\mathrm{F}$ & $\mathrm{P}$ \\
\hline Espèce & 2 & 39,68 & $<0,0001$ & 2 & 98,19 & $<0,0001$ & 146,28 & $<0,0001$ \\
Traitement & 3 & 300,5 & $<0,0001$ & 3 & 63,38 & $<0,0001$ & 174,95 & $<0,0001$ \\
Espèces $\times$ traitement & 6 & 20,41 & $<0,0001$ & 6 & 5,87 & 0,0006 & 23,31 & $<0,0001$ \\
\hline
\end{tabular}

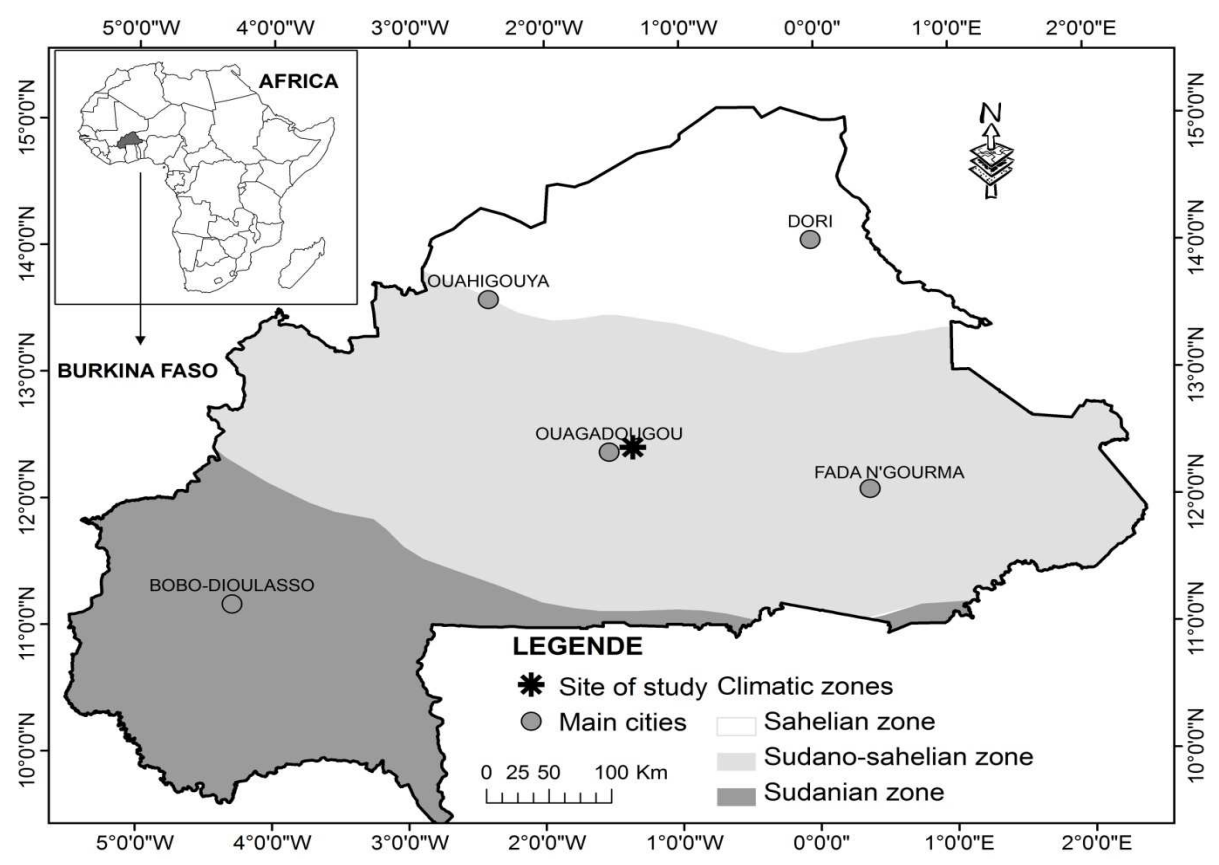

Figure 1 : Localisation géographique du site d'étude 


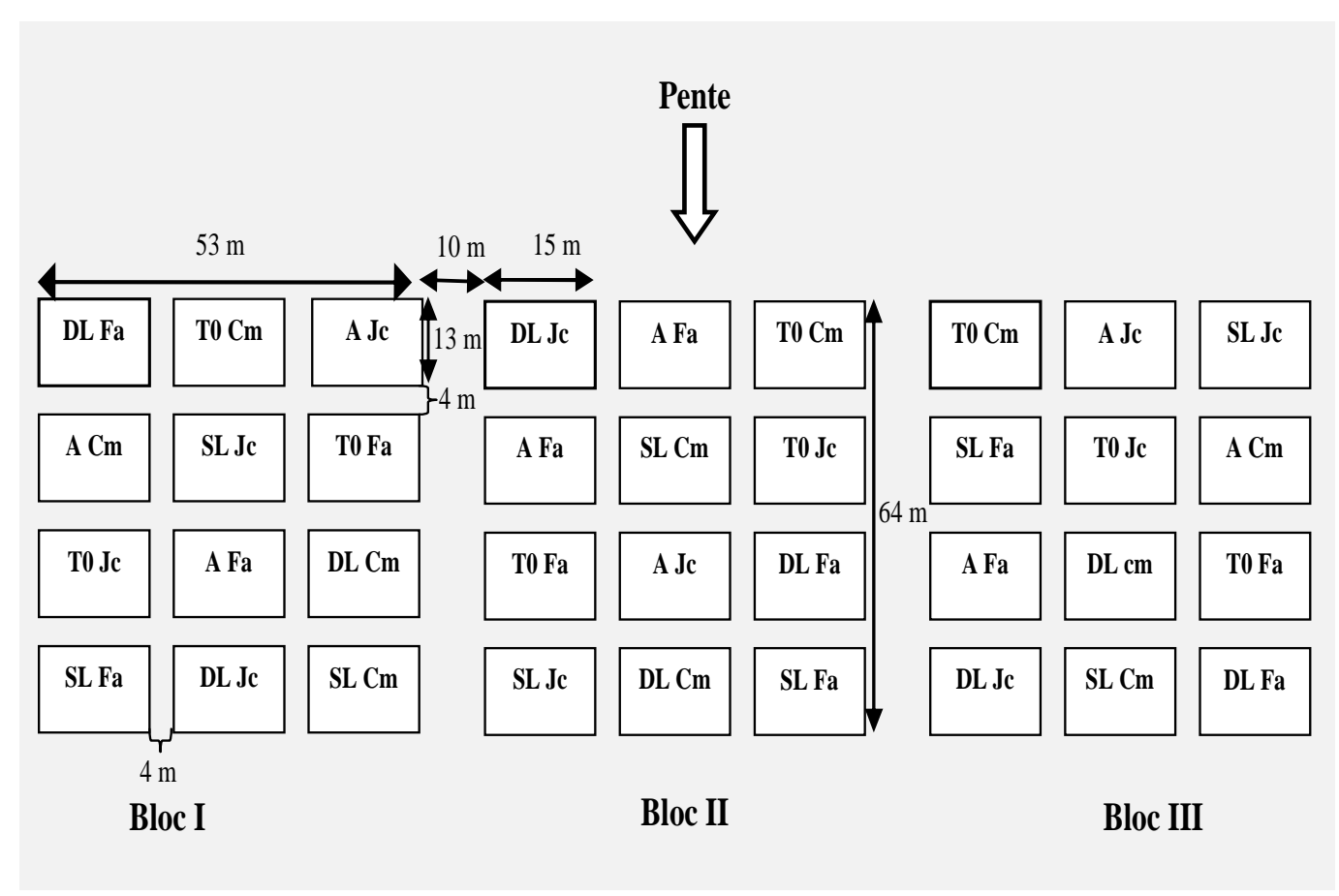

Figure 2 : Schéma du dispositif expérimental.

Traitements: $\mathrm{T} 0=$ témoin; $\mathrm{A}=$ amendement, $\mathrm{DL}=$ demi-lunes; $\mathrm{SL}=$ sous-solage; Espèces: $\mathrm{Cm}=C$. micranthum $; \mathrm{Fa}=F$. albida et $\mathrm{Jc}=J$. curcas

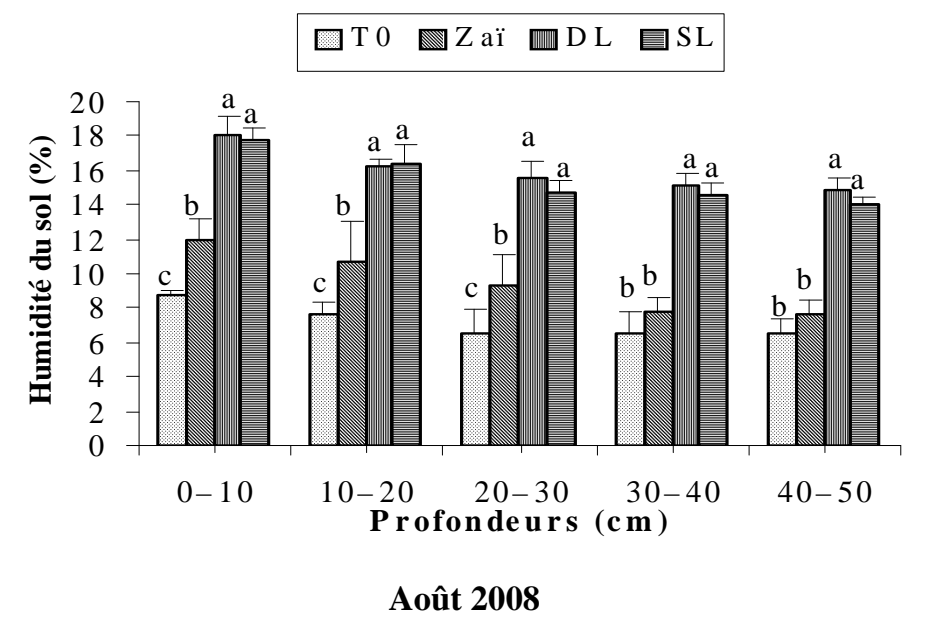




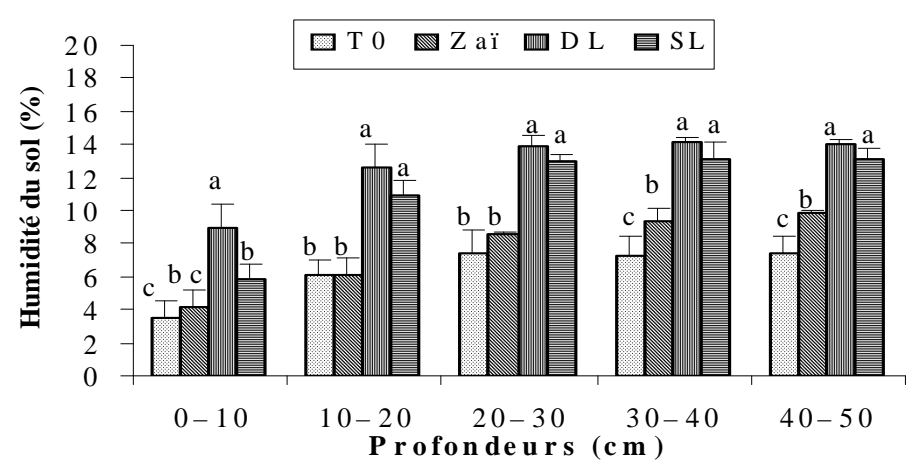

Octobre 2008

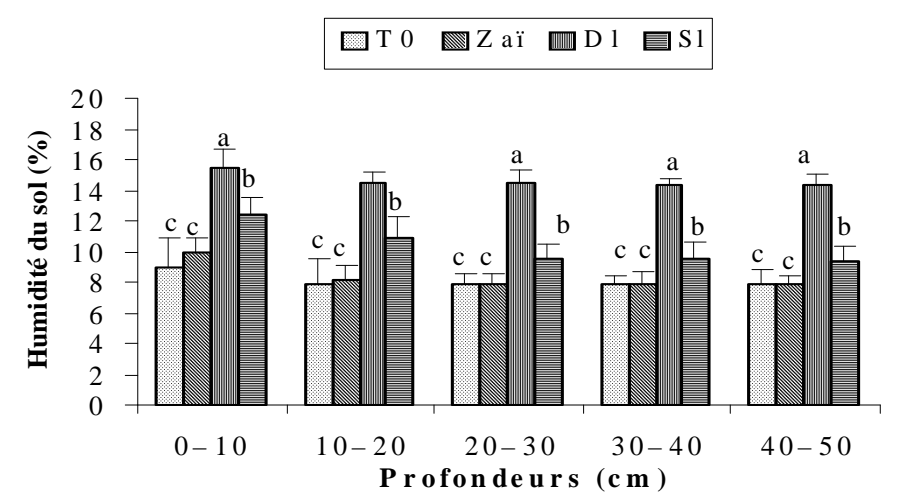

Août 2009

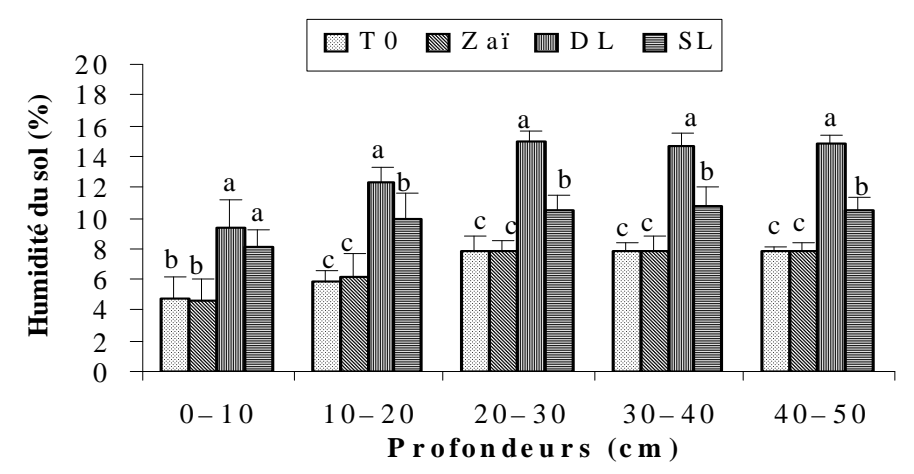

Octobre 2009

Figure 3 : Effets des aménagements sur l'humidité du sol (\%) suivant les niveaux de profondeurs en 2008 et 2009.

Les valeurs avec les mêmes lettres ne sont pas statistiquement différents au seuil de 5\% selon le test de Tukey HSD: T0 = témoin; $\mathrm{A}=$ amendement DL = demi-lunes; $\mathrm{SL}=$ Sous-solage $;$ Les barres sont des écarts-types.

T0 = témoin; $\mathrm{A}=$ amendement, $\mathrm{DL}=$ demi-lunes; $\mathrm{SL}=$ sous-solage $;$ Les valeurs utilisées sont des moyennes et les lettres dénotent la significativité au seuil de 5\% selon le test Tukey HSD. Les barres sont des écarts-types. 


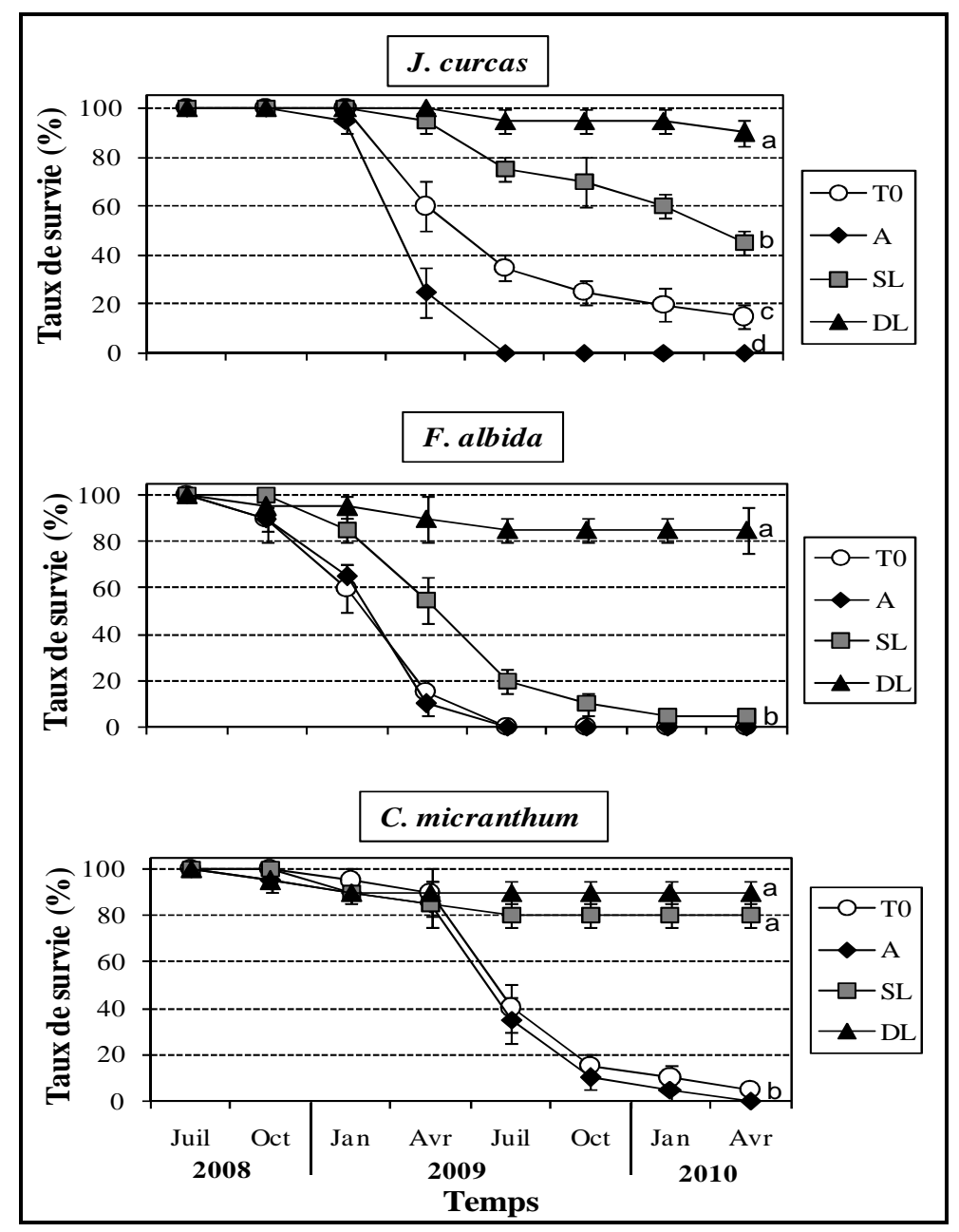

Figure 4 : Effets des aménagements sur la variation temporelle des taux de survie des espèces. Les valeurs utilisées sont des moyennes et les lettres à la fin des courbes dénotent la significativité au seuil de 5\% selon le test Tukey HSD. Les barres sont des écarts-types.

\section{DISCUSSION}

Le problème de la dégradation des sols constitue une grande menace pour la zone tropicale. La disponibilité d'une végétation permet de rétablir l'équilibre de ces sols dégradés et de déclencher un mécanisme d'évolution progressive (Lal, 2004). Cependant, l'eau joue un rôle fondamental dans le développement des végétaux. La variation gravimétrique de l'humidité dans le sol est contrôlée par plusieurs processus hydrologiques régis par l'infiltration (Ambouta et al., 1996) et l'évapotranspiration. Les différences observées sont surtout régies par les infiltrations pourtant limitées par les croûtes de surface et la compaction des sols (Zougmoré et al., 2003). La destruction de la croûte par le décapage de l'horizon de surface améliore ne serait ce que temporairement la porosité du sol, puis l'infiltration de l'eau (Casenave et Valentin, 1992). Les variations inter-aménagements s'expliqueraient donc par les différences de capacité de stockage hydrique de ces aménagements. Les DL à cause de leur grande capacité de capture des eaux de surface (Zougmoré et al., 2003), et le SL ont enregistré les plus forts taux d'humidité à la première année. Toutefois à la 


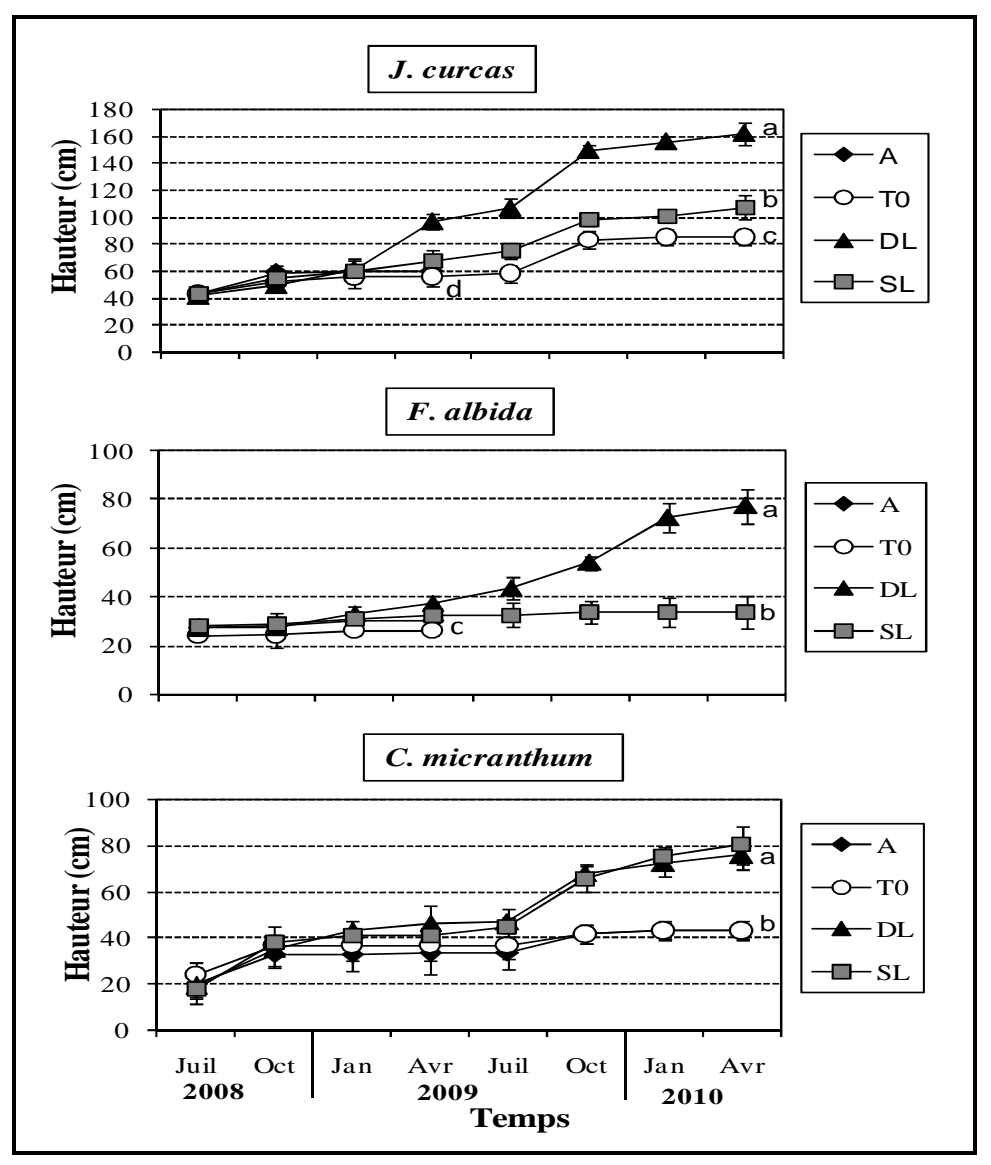

Figure 5 : Effets des aménagements sur l'évolution temporelle de la hauteur des espèces. Les valeurs sont des moyennes et les lettres à la fin des courbes dénotent la significativité au seuil de $5 \%$ selon le test Tukey HSD. Les barres sont des écarts-types.

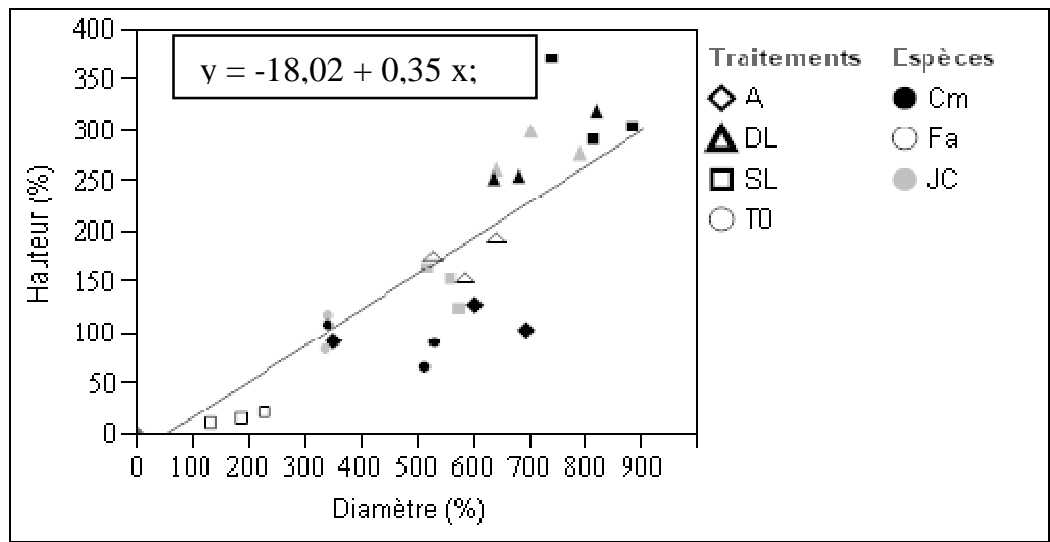

Figure 6 : Corrélation entre les taux de croissance en hauteur et en diamètre suivant les traitements et les espèces à la fin de l'essai.

Traitements: $\mathrm{T} 0=$ témoin; $\mathrm{A}=$ amendement, $\mathrm{DL}=$ demi-lunes; $\mathrm{SL}=$ sous-solage; Espèces: $\mathrm{Cm}=C$. micranthum; $\mathrm{Fa}=F$. albida et $\mathrm{Jc}=J$. curcas. 
deuxième année, pendant que les sillons de SL subissaient des désorganisations structurales dues à l'érosion, les buttes de terres des DL demeuraient toujours bien en place du fait de leur disposition semi-perméable. Cela expliquerait les différences entre les deux aménagements; pendant que l'humidité baissait dans le SL, les DL présentaient par une humidité similaire à la première année surtout en octobre.

Les taux de croissance et de survie constituent des paramètres essentiels indiquant la réussite d'une opération d'afforestation. Différents facteurs du milieu influencent la croissance et la survie des plants en milieu aride. Les facteurs les plus importants sont la compacité, la fertilité et l'humidité du sol (Hien, 2000; Hiernaux et Le Houérou, 2006). Les différences de croissance et de survie des espèces selon les traitements sont donc liées aux conditions hydriques et nutritionnelles induit par ces derniers. Pendant la saison pluvieuse, les aménagements de DL et SL avaient les meilleurs taux d'humidité. Ainsi ces aménagements ont produit les plus forts accroissements chez $C$. micranthum. Chez J. curcas, en contraste avec les tendances de l'humidité, l'accroissement était plus élevé dans l'aménagement A; aussi la similarité entre les DL et le témoin suggère que l'eau n'était pas un facteur limitant pendant ce temps pour cette espèce qui est une plante succulente. Lorsque l'humidité du sol n'est pas un facteur limitant, la croissance est contrôlée par la nutrition minérale (Roose, 2004; Hiernaux et Le Houérou, 2006); d'où les meilleurs accroissements obtenus dans le traitement A. La non influence des traitements pendant ce temps chez $F$. albida peut être lié à sa phénologie inversée ou à un problème de nodulation dû aux caractéristiques du milieu. Après la saison pluvieuse, l'humidité du sol devient le facteur principal influençant la croissance et la survie des plants surtout en milieu aride. Son déficit entraîne la perturbation des mécanismes physiologiques et provoque un stress hydrique et une mortalité s'il perdure (Hien, 2000). Dans ces conditions, pour continuer à extraire l'eau du sol, les plantes doivent faire face aux variations journalières, mensuelles et annuelles pendant la saison sèche (Gebrekirstos et al., 2006). L'aptitude d'une plante à absorber l'eau dans ces milieux est affectée par la profondeur et la distribution de son système racinaire (Grossinickle, 2005). En effet, les trois espèces étudiées se caractérisent par un enracinement à la fois pivotant et traçant. Toutefois les facteurs tels la structure, la texture, la compacité et le niveau d'humidité du sol influent sur le type d'enracinement pour une espèce donnée. Ainsi, les différences de comportement des plants observées suivant les aménagements pourraient s'expliquer en partie par les modifications de conditions édaphiques qu'ils occasionnent et surtout l'humidité du sol. Les taux d'humidité étaient toujours élevés surtout en profondeur dans les DL, dans une moindre mesure dans le SL et faibles dans les T0 et les A; d'où les meilleurs performances des espèces dans les DL suivi par le SL. La mauvaise performance de $F$. albida hormis dans les DL serait liée à la nature argileuse et compacte des sols du site. En effet, sur sol sableux, le pivot se développe en profondeur alors que sur sol argileux compact, le système racinaire reste traçant et superficiel (Alexandre et Ouédraogo, 1993) exposant ainsi la plante aux stress hydriques surtout en saison sèche. La forte mortalité de $J$. curcas dans les parcelles amendées (A) est imputable aux attaques des termites (Kumar et al., 2008).

Dans l'ensemble, il apparaît que l'aménagement en demi-lune suivi de celui de sous-solage, constituent les ouvrages qui permettent un important accroissement et de meilleurs taux de survie (> 70\%) chez les trois espèces. Ces observations sont conformes à des résultats déjà obtenus en zone soudano- 
sahélienne du Burkina Faso avec d'autres espèces (Ganaba, 2005; Zoubga, 2002). Toutefois les valeurs plus élevées en hauteur et taux de survie de notre essai sont attribuables au statut de protection dont il a bénéficié.

Les résultats escomptés par cette étude montrent une influence significative des techniques étudiées sur l'amélioration des conditions hydriques des sols ainsi que sur la croissance et les taux de survie des espèces. Excepté la similarité avec l'aménagement de sous-solage chez $C$. micranthum, l'aménagement de demi-lunes a donné les meilleures performances chez toutes les espèces. Les plantations de ces espèces en combinaison avec les demi-lunes permettraient donc de valoriser les terres dégradées et dénudées qui ne sont pas utilisées pour l'agriculture vivrière. Son utilisation à grande échelle permettra la multiplication des espèces retenues afin de garantir la disponibilité d'une végétation rétablissant l'équilibre de ces sols dégradés.

Toutefois, des recherches complémentaires sont nécessaires afin de pouvoir évaluer l'effet de l'amendement en fumure organique sur la croissance et la productivité des espèces dans les aménagements purement physiques (DL et SL). Aussi, les essais futurs devraient considérer des mesures de protection contre les termites.

\section{Conclusion}

En somme, les techniques de restauration étudiées améliorent de façon significative les taux de croissance et de survie des espèces étudiées. A la fin de l'essai, l'aménagement en demi-lunes apparaît donc comme la méthode appropriée garantissant la régénération artificielle de ces espèces sur les zipellés. Toutefois, il s'agit d'une technique physiquement exigeante et son utilisation pertinente pour atténuer la dégradation des sols dépend de son adoption par les bénéficiaires et de la disposition des moyens financiers pour sa mise en œuvre. Ainsi, il faudra développer des initiatives décisives et adaptées au contexte avec des mesures d'accompagnement afin d'envisager une appropriation éventuelle et une responsabilisation plus accentuée. Une subvention de la part des organisations non gouvernementales (ONG) de développement et des gouvernements est donc nécessaire pour permettre la réalisation des différentes activités.

\section{REMERCIEMENTS}

Cette étude a été conduite grâce au support financier du projet «Sustainable Use of Natural Vegetation in west africa » (SUNEU FP6 031685). C'est une occasion pour les auteurs de manifester leur gratitude à l'égard de ce projet. Les auteurs remercient également les évaluateurs pour les différents amendements apportés sur le manuscrit.

\section{REFERENCES}

Alexandre DY, Ouédraogo SJ. 1993. Variations de la morphologie racinaire de Faidherbia albida en relation avec le sol et effets agronomiques de l'arbre. In Faidherbia albida dans les Zones Tropicales Semi-arides d'Afrique de l'Ouest, Vandenbeldt RJ, Renard C (eds). A.P. 502 324: Patancheru, Inde; 107-110.

Ambouta KMJ, Valentin C, Laverdière MR 1996. Jachères et croûtes d'érosion au Sahel. Sécheresse, 7: 269-275.

Assogbadjo AE, Amadji G, Glèlè Kakaï R, Mama A, Sinsin B, Van Damme P. 2009. Evaluation écologique et ethnobotanique de Jatropha curcas L. au Benin. Int. J. Biol. Chem. Sci., 3(5): 1065-1077.

Bationo A, Hartemink A, Lungu O, Naimi M, Okoth P, Smaling E, Thiombiano L. 2006. African Soils : Their productivity and profitability of fertilizer use. Background 
Paper, Africa Fertilizer Summit 2006, 913 June, Abuja, p. 25.

Bellefontaine R, Et-tobi M, Mhirit O. 2005. Les ligneux urbains et agrosylvopastoraux des zones sèches et arides à faible couvert ligneux. Sécheresse, 16(4): 306-308.

Casenave A, Valentin C. A runoff capability classification system based on surface features criteria in the arid and semi-arid areas of West Africa. J. Hydrol., 130: 231-249.

Dixon RK, Andrasko KJ, Sussman FG, Lavinson MA, Trexler MC, Vinson TS. 1993. Forest sector carbon offset projects: near- term opportunities to mitigate greenhouse gas emissions. Water Air and Soil Pollution, 70: 561-577.

FAO 2006. Word Reference Base of soil. World soil resources reports, 103: 127.

FEM-FIDA. 2002. S'attaquer à la Dégradation des Terres. FEM-FIDA: Rome ; p. 9.

Fontes J, Guinko S. 1995. Carte de la végétation et de l'occupation du Burkina Faso. Note explicative. Toulouse: Ministère de la coopération française, $\mathrm{p}$. 53.

Ganaba S. 2005. Impact des aménagements de conservation des eaux et des sols sur la régénération des ressources ligneuses en zone sahélienne et nord soudanienne du Burkina Faso. Vertigo, 6: 126-140.

Ganaba S, Bayala J,Yaméogo KM, Yaméogo JT, Ouadba J-M. 2006. Impact des aménagements anti-érosifs sur la phénologie, la transpiration et la productivité fruitière ligneuse en zone subsahélienne du Burkina Faso. Etudes sur la Flore. Vég. Burkina Faso, 10: 4962.

Gebrekirstos A, Teketay D, Fetene M, Mitlöhner R. 2006. Adaptation of five cooccurring tree and shrub species to water stress and its implications in restoration of degraded lands. Forest Ecology and Management, 229: 259-267.

Grossinickle SC. 2005. Importance of root growth in overcoming planting stress. New Forests, 30: 273-294.

Hien FG. 1995. La régénération de l'espace sylvo-pastoral au Sahel: Une étude de l'effet des mesures de conservation des eaux et des sols au Burkina Faso. Document sur la gestion des ressources tropicales $\mathrm{n}^{\circ} 7$, Université de Wageningen, p. 194.

Hien MB. 2000. Etude des causes de mortalité de Pterocarpus lucens en zone subsahélienne du Burkina Faso. Mémoire Ingénieur des Eaux \& Forêts, Univ. Polytech. de Bobo, p. 87.

Hiernaux P, Le Houérou HN. 2006. Les parcours du sahel. Sécheresse, 17(1-2): 51-71.

Kumar GP, Yadav SK, Thawale PR, Singh S K, Juwarkar AA. 2008. Growth of Jatropha curcas on heavy metal contaminated soil amended with insdustrial wastes and Azotobacter- A green house study. Bioresource technology, 99: 2078-2082.

Lal R. 2004. Carbon Sequestration in Dryland Ecosystems. Environmental Management, 33: 528-544.

Pule-Meuelenberg F, Dakora FD. 2005. Assessing the biological potential of N2fixing Leguminosae in Botswana for increased crop yields and commercial exploitation. African Journal of Biotechnology, 6(4): 325-334.

Roose E. 2004. Evolution historique des stratégies de lutte antiérosive vers la gestion conservatoire de l'eau, de la biomasse et de la fertilité des sols. Sécheresse, 15(1): 9-18.

SAS Institute. 2007. Statistics and graphics guide. Cary, SAS Institute Inc., NC.

Thiombiano A. 2005. Les Combretaceae du Burkina Faso: taxonomie, écologie, 
dynamique et régénération des espèces. thèse de doctorat, Univ. de Ouagadougou, Burkina Faso, p. 290.

Thiombiano L, Tourino-Soto I. 2007. Status and Trends in Land Degradation in Africa. In Climate and Land Degradation, Environmental Science and Engineering, Sivakuma M.V.K., Ndang'ui N. (Eds.). Springer: 39-53.

Traore S, Thiombiano L, Rasolomdimby Millogo J, Guinko S. 2007. Carbon and nitrogen enhancement in cambisols and vertisols by Acacia spp. in eastern Burkina Faso: relation to soil respiration and microbial biomass. Applied Soil Ecology, 35: 660-669.

UNDP-GEF. 2004. Reclaiming the Land Sustaining Livelihoods - Lessons for the Futur. UNDP/GEF: NY, USA; 20.

Zoubga TS. 2002. Etude de l'impact du travail du sol des charrues Delphino et Tréno sur le sol et la végétation des terres dégradées du Soum (Burkina Faso). Mémoire Inspecteur des Eaux \& Forêts, ENEF, p. 90.

Zougmoré R, Ouattara K, Mando A, Ouattara B. 2004. Rôle des nutriments dans le succès des techniques de conservation des eaux et des sols (cordons pierreux, bandes enherbées, zaï et demi-lunes) au Burkina Faso. Sécheresse, 15: 41-48.

Zougmoré R, Zida Z, Kambou NF. 2003. Role of nutrient amendments in the success of half-moon soil and water conservation practice in semiarid Burkina Faso. Soil \& Tillage Research, 71: 143-149. 